Cahiers de Narratologie

Analyse et théorie narratives

$7 \mid 1996$

Mélanges espace \& temps

\title{
Le temps du récit cinématographique
}

Sophie Velasco

\section{OpenEdition}

Journals

Édition électronique

URL : https://journals.openedition.org/narratologie/11799

DOI : 10.4000/narratologie. 11799

ISSN : 1765-307X

Éditeur

LIRCES

\section{Édition imprimée}

Date de publication : 1 janvier 1996

Pagination : 115-134

ISSN : 0993-8516

\section{Référence électronique}

Sophie Velasco, "Le temps du récit cinématographique », Cahiers de Narratologie [En ligne], 7 | 1996,

mis en ligne le 03 mai 2021, consulté le 05 mai 2021. URL : http://journals.openedition.org/

narratologie/11799; DOI : https://doi.org/10.4000/narratologie.11799

Ce document a été généré automatiquement le 5 mai 2021.

Article L.111-1 du Code de la propriété intellectuelle. 


\title{
Le temps du récit cinématographique
}

\author{
Sophie Velasco
}

1 Afin d'aider le lecteur à mieux comprendre notre choix, nous avons jugé utile d'expliquer brièvement de quoi il est question dans Crónica ... et de quelle façon les événements nous sont présentés.

2 Un jeune homme fortuné arrive dans un petit village colombien où va se dérouler toute l'histoire : il se prénomme Bayardo San Roman. Il tombe amoureux d'une jeune fille, Àngela Vicario, et la demande en mariage. Àngela, qui n'éprouve aucun sentiment amoureux envers Bayardo, s'oppose dans un premier temps à cette union. Puis sous la pression exercée par sa famille, elle sera contrainte de l'accepter. Le mariage est donc célébré, mais dès le lendemain, Bayardo doit répudier sa jeune épouse qui n'était pas vierge. C'est alors que les frères Vicario obligent leur sœur à dénoncer le responsable de son déshonneur. Celle-ci accuse un jeune homme du village, Santiago Nasar. Par conséquent, les frères Vicario se croient dans l'obligation de venger l'honneur de leur famille en tuant ce dernier.

327 ans après ce crime, Cristóbal Bedoya, le meilleur ami de Santiago Nasar, qui a alors une cinquantaine d'années, ne parvient toujours pas à comprendre comment ce drame n'a pu être évité, alors que le village entier connaissait l'intention meurtrière des frères Vicario. C'est pour cette raison qu'il revient dans son village d'origine, essayant de faire resurgir des souvenirs enfouis au plus profond de sa mémoire. Il espère ainsi se remémorer, afin de mieux le comprendre, ce drame qui l'a profondément marqué.

4 Notre film ne respecte pas l'ordre chronologique des événements. Il débute par le retour au village de Cristóbal Bedoya 27 ans après le crime, et c'est par ses souvenirs que nous allons retourner dans le passé.

5 Comme nous pouvons le voir, notre récit cinématographique est le récit du souvenir. Par conséquent, il nous a semblé particulièrement intéressant d'analyser le temps. 
6 Nous tenons à rappeler que le récit est une séquence doublement temporelle. En effet, un récit cinématographique, de même qu'un récit littéraire, met en jeu deux temporalités :

7 d'une part, celle des événements racontés, soit le temps de la fiction; d'autre part, celle qui tient à l'acte narratif lui-même, soit le temps de la narration ${ }^{1}$.

Le temps de la fiction est l'espace temporel délimité par les dates ou les informants qui annoncent le début et la fin de l'histoire ; il est linéaire et inexorablement orienté vers le futur.

9 Le temps de la narration est le temps nécessaire à l'énonciation de la fiction.

10 Dans l'univers construit par la fiction, la diégèse ${ }^{2}$, un événement peut se définir par la place qu'il occupe dans la chronologie supposée de l'histoire, par sa durée, par le nombre de fois où il intervient ${ }^{3}$.

11 Le narrateur n'est pas obligé de respecter cette temporalité diégétique pour faire connaître cet événement : il peut choisir de commencer par un fait plutôt que par un autre (c'est la question de l'ordre), de le raconter longuement ou au contraire brièvement (c'est la question de la durée), de l'évoquer à une ou plusieurs reprises (c'est la question de la fréquence). Son récit a donc une temporalité spécifique, différente de celle de l'histoire ${ }^{4}$.

\section{A / L'ORDRE}

Dans un premier temps, nous étudierons la question de l'ordre, en confrontant la succession des événements supposée par la diégèse, à l'ordre de leur apparition dans le récit cinématographique.

13 Ces distorsions qui existent entre la chaîne événementielle supposée réelle de la fiction (temps de l'énoncé), et la chaîne événementielle telle qu'elle apparaît dans le discours (temps de l'énonciation) donnent le temps narrés.

\section{1 - Temps de l'énoncé}

14 Étudions plus concrètement le temps de l'énoncé dans Crónica de una muerte anunciada. En premier lieu, nous allons voir quel est l'ordre de succession des événements dans l'histoire.

15 Tout d'abord, le premier élément de la diégèse est l'arrivée d'un jeune homme, Bayardo San Román, dans le village où se déroulera toute l'histoire.

16 Nous assistons ensuite, aux événements suivants :

17 - ses rencontres avec Ángela Vicario, une jeune fille dont il tombe amoureux

18 - l'acquisition par Bayardo de la maison d'un vieil homme, le veuf Xious

19 - le mariage d'Ángela et Bayardo

20 - la nuit de noces et la répudiation

21 - l'aveu d'Ángela accusant Santiago Nasar

22 - la préparation de la vengeance par les frères Vicario

23 - l'assassinat de Santiago Nasar 
24 - le procès

25 - le départ de Bayardo

26 - le retour au village de Cristóbal Bedoya chargé de s'occuper de l'hôpital

27 - les retrouvailles d'Ángela et Bayardo (dénouement)

28 De plus, nous disposons d'une série d'informants temporels qui nous permettent de dater certains événements de façon plus ou moins précise.

29 Commençons par l'événement principal de notre film: la mort de Santiago Nasar. Cristóbal Bedoya nous informe à la séquence 6 qu'elle a eu lieu trois ans après celle de son père :

"Pero él parecía ser feliz con su padre, hasta que éste murió de repente tres años antes $[. .] "$.6 .

Or le père de Santiago, Ibrahim Nasar, est mort en 1940, comme nous l'apprenons lorsque Cristóbal Bedoya se recueille au cimetière. Nous avons une focalisation sur la tombe d'Ibrahim Nasar. Nous déduisons de ces deux informants que le crime a eu lieu en 1943.

31 D'autre part, alors que nous ignorons le mois et le jour exact de la mort de Santiago, nous découvrons qu'elle est survenue un lundi, ["el lunes de su muerte"7], informant donné par Cristóbal Bedoya à la séquence 6.

Nous pouvons également reconstituer l'heure du crime: après avoir vu l'évêque, Santiago quitte le quai à 6 h25 du matin. En effet, il demande l'heure à son ami Bedoya qui lui répond, à la séquence 14 :

"Las seis y veinticinco"s

Or il meurt quelques instants plus tard, soit vers $6 \mathrm{~h} 30$.

Le crime a donc eu lieu un lundi de 1943 vers 6h30 du matin. célébré le dimanche. Un autre événement que nous pouvons dater par rapport au crime est l'arrivée au village de Bayardo San Román. Cristóbal Bedoya dit aux séquences 17 et 18 :

"Seis meses antes de que mataran a Santiago Nasar, llegó a nuestro pueblo un extranjero... Se llamaba Bayardo San Román"9 Bayardo est donc arrivé six mois avant le crime.

Enfin, le retour de Cristóbal Bedoya au village, qui revient afin de s'occuper de l'hôpital et essayer de "recomposer [...] le miroir cassé de la mémoire », a lieu 27 ans après le crime, soit en 1970. Il dit en effet à la première séquence :

"He vuelto después de 27 años a este pueblo olvidado para tratar de recomponer con tantas astillas dispersas, el espejo roto de la memoria"10

\section{2 - Temps de l'énonciation}

Nous nous intéresserons plus particulièrement à l'ordre d'apparition des événements dans le récit cinématographique.

Comme nous l'avons d'ores et déjà remarqué, la chaîne événementielle telle qu'elle apparaît dans le récit diffère totalement de la chaîne événementielle supposée réelle de la fiction. D'où l'intérêt d'analyser le temps narré. 


\section{a / Récit premier / récit(s) second(s)}

Déterminons tout d'abord le récit premier du (ou des) récits second(s) dans notre film.

La séquence ${ }^{11}$ qui ouvre le film nous présente un homme d'âge mûr (nous saurons plus tard qu'il s'agit de Cristóbal Bedoya), qui revient dans son village d'origine 27 ans après un événement qu'il va essayer de se remémorer. Par rapport à cette première séquence [0], nous serons tour à tour rejetés en arrière (évocation du crime 27 ans auparavant $[B]$ ), ramenés à notre point de départ, renvoyés à nouveau au passé (évocation de l'arrivée de Bayardo au village six mois avant le crime [A]), ainsi de suite.

Dans une œuvre littéraire ou filmique, il existe donc un écoulement du temps, que nous figurerons par un déplacement du point 0 , de gauche à droite, sur l'axe $x-y$, et que nous appellerons récit premier ${ }^{12}$.

4 Ce que nous pouvons représenter de la façon suivante :

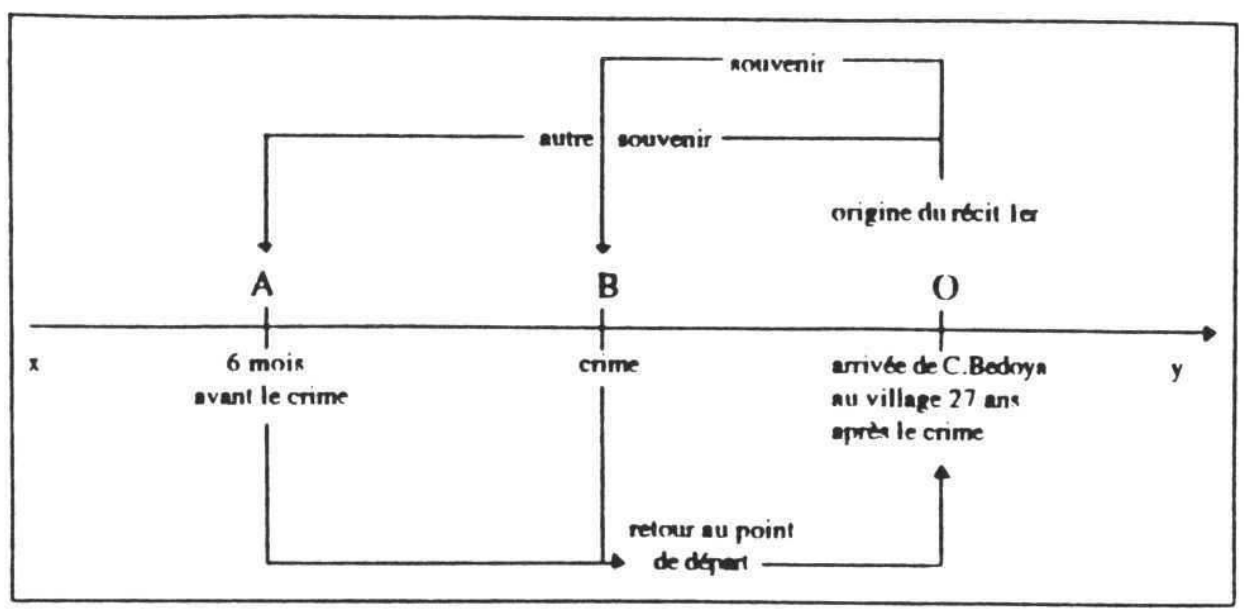

Comme nous le voyons, le point $O$ est l'instant à partir duquel s'organise le récit, en nous faisant voyager dans le temps. Par rapport à cette référence, les points $\mathrm{A}$ et $\mathrm{B}$ sont rétrospectifs puisqu'ils évoquent des événements intervenus auparavant dans la diégèse.

46 L'évocation a posteriori d'un événement antérieur au moment de l'histoire où nous nous trouvons [0], Genette l'appelle analepse (du grec -lapse signifiant prendre, et anasignifiant après), par opposition à prolepse (intervention d'un événement qui arrive avant sa place normale dans la chronologie).

Les analepses et les prolepses sont deux types d'anachronies narratives que Genette définit comme « les différentes formes de discordance entre l'ordre de l'histoire et celui du récit $»^{13}$.

4 Dans Crónica..., nous appellerons donc récit premier le récit de Cristóbal Bedoya qui revient dans son village 27 ans après, car c'est à partir de lui que nous allons voyager dans le passé.

D'autre part, selon Genette, toute anachronie constitue par rapport au récit dans lequel elle s'insère, un récit temporellement second ${ }^{14}$. Dans notre film, la série d'analepses subordonnées au récit premier, et que nous allons étudier, constituent donc des récits seconds. 


\section{b / Les analepses}

Avant d'analyser cas par cas les analepses présentes dans notre film, nous allons voir précisément quelles sont les caractéristiques à étudier.

Nous parlerons tout d'abord de portée de l'analepse, qui est la distance temporelle plus ou moins grande entre la séquence anachronique et le moment de l'histoire où le récit s'est interrompu pour lui faire place.

Ensuite, nous étudierons son amplitude, qui est la durée d'histoire plus ou moins longue qu'elle couvre ${ }^{15}$.

D'autre part. Genette sépare les analepses en deux grandes catégories :

- celles qu'il qualifie d'externes, c'est-à-dire, celles dont toute l'amplitude reste extérieure à celle du récit premier. Elles ne risquent à aucun moment d'interférer avec le récit premier, qu'elles ont seulement pour fonction de compléter.

- celles qu'il qualifie d'internes, dont le champ temporel est compris dans celui du récit premier.

Genette propose de diviser à leur tour les analepses internes en deux sous-catégories :

- les analepses internes hétérodiégétiques, c'est-à-dire portant sur une ligne d'histoire, et donc un contenu diégétique, différents de celui du récit premier, soit très classiquement un personnage nouvellement introduit et dont le narrateur veut éclairer les antécédents.

- les analepses internes homodiégétiques, c'est-à-dire qui portent sur la même ligne d'action que le récit premier ${ }^{16}$.

Nous nous proposons d'analyser les différentes analepses du film.

\section{$\alpha /$ Première analepse}

Elle se situe vers le tout début du film. Son point de départ se trouve dans le récit premier, lorsque Cristóbal Bedoya se recueille sur la tombe de son ami, Santiago Nasar, mort 27 ans auparavant. Nous observons un gros plan sur la photo du défunt, et de là part l'analepse.

Nous y voyons Santiago se réveillant brusquement à l'aube après un cauchemar. Il discute un moment avec sa mère, descend à la cuisine où se trouvent la cuisinière et sa fille Divina Flor, puis se rend sur le quai afin d'accueillir l'évêque. Ensuite, il prend congé de ses amis, Cristóbal Bedoya et Margot, à 6h25, en promettant à celle-ci de la rejoindre chez elle pour y prendre le petit-déjeuner. Cette dernière apprend par des jeunes filles du village que les frères Vicario veulent tuer Santiago Nasar pour avoir déshonoré leur sœur Ángela. Quelques minutes plus tard, vers 6h30, des cris nous annoncent à la séquence 16 que le crime a eu lieu :

"Lo mataron... Esta muerto"17.

Nous apprendrons plus tard dans le film que Santiago Nasar s'est réveillé vers $5 \mathrm{~h} 30$. Il dit en effet à la cuisinière, avant de se coucher, à la séquence 45 :

"Mándame a Divina Flor para que me despierte a las cinco y media [...]"18.

La portée de cette analepse est très grande puisqu'elle est de 27 ans. En revanche, son amplitude est très petite puisqu'elle est d'une heure environ.

D'autre part, elle reste extérieure à celle du récit premier. Nous pouvons donc qualifier cette première analepse d'externe. 
Le but de cette première analepse est donc de nous présenter la victime, les assassins et le mobile du crime. Donc dès le début du film, nous savons qui est tué, par qui, et pourquoi : il n'y a plus aucun suspense.

\section{$\beta /$ Seconde analepse}

61 Elle se situe environ au premier quart du film. Elle s'insère dans le récit premier, au moment où Cristóbal Bedoya, assis au bord du fleuve, se souvient de l'arrivée d'un étranger au village, six mois avant le crime (séquence 17):

"6 meses antes de que mataran a Santiago Nasar, llegó a nuestro pueblo un extranjero" 19 .

62 Nous voyons le fleuve couler derrière lui : voilà le point de départ de cette $2^{\text {ème }}$ analepse, qui comporte le récit de l'arrivée au, village de Bayardo San Román, ses efforts pour attirer la sympathie d'Ángela, une jeune fille dont il s'est épris, leur mariage, la répudiation d'Ángela, l'aveu de la jeune femme qui rend Santiago Nasar coupable de son déshonneur, la préparation de la vengeance par les frères Vicario, la poursuite sur la place des frères Vicario qui cherchent à tuer Santiago, et la fuite de ce dernier vers chez lui, jusqu'à ce qu'on lui «ferme la porte au nez», la réaction de Bayardo San Román qui noie son chagrin dans l'alcool jusqu'à ce que sa famille vienne le chercher, la nostalgie d'Ángela qui retourne dans la maison qui fut la sienne l'espace d'une nuit, et qui pendant des années, écrira des lettres d'amour à Bayardo, et enfin, la rencontre entre Ángela et Cristóbal Bedoya, lorsque ce dernier revient au village 27 ans après le crime.

63 La portée de cette seconde analepse est de 27 ans et demi, puisqu'elle remonte à 6 mois avant le crime. Son amplitude est également de 27 ans et demi, puisque nous venons de voir que ce récit second rejoint le récit premier. La portée de l'analepse est donc égale à son amplitude. Le point de portée de cette analepse se situe à l'extérieur du champ temporel du récit premier. Elle est donc externe par son début. Cependant, une partie de son champ temporel est comprise dans celui du récit premier. Elle est donc interne par sa fin. Genette qualifie ce type d'analepse de mixte:

«La classe mixte est en fait déterminée par une caractéristique d'amplitude, puisqu'il s'agit d'analepses externes qui se prolongent jusqu'à rejoindre et dépasser le point de départ du récit premier ${ }^{20}$

64 Cette seconde analepse nous permet de comprendre quel rôle a joué la fatalité dans le déroulement des événements. Le but est donc de mettre en relief l'importance fondamentale de la fatalité.

\section{$\gamma /$ Troisième analepse}

Elle se situe à la moitié du film. Il s'agit du procès concernant le crime de Santiago Nasar. Son point de départ se trouve au moment où Cristóbal Bedoya se rend à l'édifice colonial et parvient à réunir plus de la moitié des feuilles éparpillées qui constituaient le dossier sur l'affaire Santiago Nasar.

Cela fait naître en lui le souvenir du procès : c'est le début de l'analepse qui s'insère elle aussi dans le récit premier. 
67 Nous y voyons tout d'abord un gros plan sur chacune des personnes présentes au procès, puis le juge interrogeant respectivement Ángela Vicario, le boucher du village, et le père Amador (le curé). (a) elle retourne, quelque temps après le crime, dans la maison que Bayardo avait achetée au veuf Xious, et se retrouve dans la chambre qui fut la sienne l'espace d'une nuit (séq. 65).

81 Si nous supposons qu'Ángela y est retournée quelques semaines après le crime, la portée de cette quatrième analepse est de quelques semaines (puisque le crime a immédiatement succédé à la nuit de noces). 

la nuit de noces fut courte si l'on considère que les jeunes mariés se sont couchés très tard après la fête, et que Bayardo ramène Ángela chez elle très tôt le matin, avant même le lever du jour.

D'autre part, le champ temporel de cette quatrième analepse est compris dans celui du récit second dans lequel elle s'insère. Cette analepse est donc interne.

Nous pouvons même la qualifier d'analepse interne homodiégétique, puisqu'elle porte sur la même ligne d'action que le récit dans lequel elle s'insère (celui que constitue la seconde analepse).

Genette fait une autre distinction en parlant de ce type d'analepse :

- les analepses complétives ou renvois, c'est-à-dire « des segments rétrospectifs qui viennent combler après coup une lacune antérieure du récit, lequel s'organise ainsi par omissions provisoires et réparations plus ou moins tardives, selon une logique narrative partiellement indépendante de l'écoulement du temps $»^{22}$.

- les analepses répétitives ou rappels, qui sont des « rappels à l'état pur volontairement choisis ou inventés pour leur caractère fortuit et banal $»^{23}$.

Selon les définitions de Genette, la quatrième analepse répond parfaitement aux critères de l'analepse complétive, puisqu'elle comble après coup une lacune antérieure du récit, celle de la nuit de noces.

\section{$\varepsilon /$ Cinquième analepse}

Il s'agit du crime proprement dit. Tout comme la quatrième analepse, celle-ci s'insère dans l'un des récits seconds, celui que constitue la seconde analepse, au moment où Cristóbal Bedoya, ayant rencontré Ángela Vicario 27 ans après le crime, lui demande la vérité, à la séquence 70 . découvre son fils mortellement blessé, gisant au sol, et qui prend conscience que c'est elle qui l'a empêché de se réfugier dans la maison familiale en fermant la porte, et enfin les frères Vicario se réfugiant à l'église et criant leur innocence.

La portée de cette analepse est de 27 ans, et son amplitude est de quelques minutes seulement.

91 D'autre part, de même que la quatrième analepse, celle-ci est interne, car son champ temporel est compris dans celui du récit second dans lequel elle s'insère (celui de la seconde analepse).

Elle porte également sur la même ligne d'action que ce dernier: elle est donc homodiégétique.

93 Enfin, elle représente un segment rétrospectif qui vient combler après coup une lacune antérieure du récit, celle du crime en question. Nous qualifierons donc cette analepse interne homodiégétique de complétive. 
94 Nous constatons que nous sommes en présence d'un récit premier, dans lequel s'insère un récit second (la seconde analepse), dans lequel s'insèrent deux récits troisièmes (les quatrième et cinquième analepses). Nous avons là l'exemple-même du récit enchâssé. Afin d'y voir plus clair, il nous a semblé nécessaire de reconstituer les schémas récapitulatifs des différents récits :

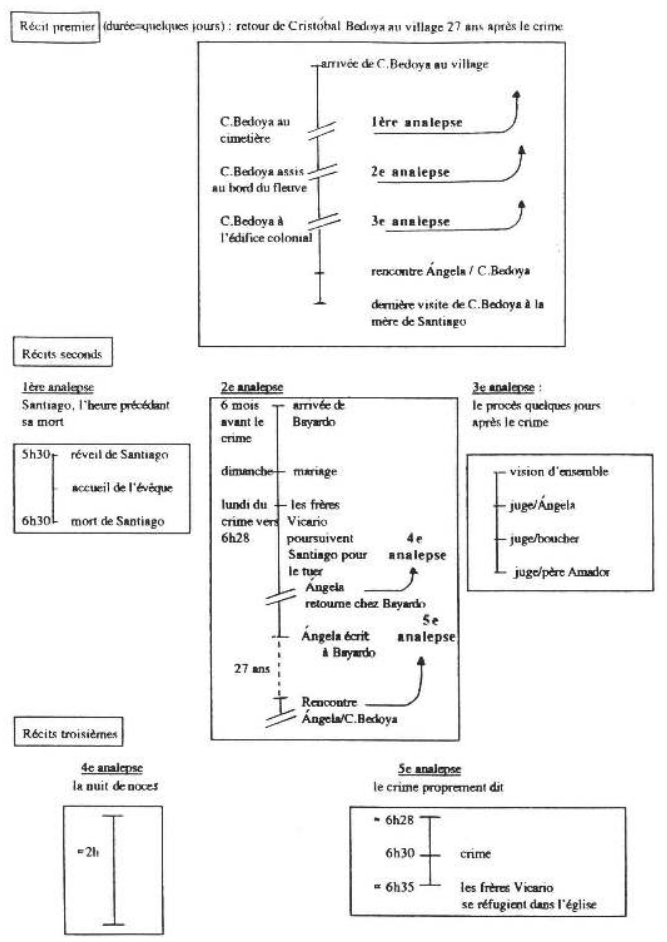

\section{c / Le dénouement}

Nous achèverons cette partie concernant l'ordre d'apparition des événements dans le récit cinématographique en parlant du dénouement de l'histoire qui occupe la fin du film.

Le récit du dénouement ne rentre pas dans le système des différents récits que nous venons d'étudier (récit premier / récits seconds / récits troisièmes). Il constitue un récit « indépendant ». Nous y voyons le retour au village de Bayardo San Román, et ses retrouvailles avec Ángela qui se jette à ses pieds en le voyant. Nous pouvons penser que Bayardo pardonne à Ángela, et ainsi s'achève l'histoire.

\section{B / LA DURÉE}

100 Il est intéressant dans l'analyse d'un film, de mettre en parallèle d'une part le temps écranique, c'est-à-dire le temps de la projection, le temps du signifiant ${ }^{25}$, d'autre part, le temps diégétique, c'est-à-dire le temps de l'histoire, le temps du signifié. 
101 Cette comparaison nous permet de percevoir des effets de rythme, que Genette appelle anisochronies. D'après lui, tout récit, qu'il soit littéraire ou cinématographique, peut se passer d'anachronies, mais pas d'anisochronies ${ }^{26}$.

En littérature, il y a pour Genette quatre principaux rythmes narratifs, qu'il schématise par les formules suivantes, où TH désigne le temps de l'histoire, et TR, le temps du récit :

- la pause, où le temps du récit est infiniment plus important que le temps de l'histoire :

TR $\infty>$ TH

L'exemple privilégié de ce cas de figure est la description qui détermine une suspension de l'histoire, une pause du récit.

- la scène, où le temps du récit équivaut au temps de l'histoire :

$\mathbf{T R}=\mathbf{T H}$

La scène dialoguée répond à ce critère.

- le sommaire où le temps du récit est plus court que le temps de l'histoire :

TR $<$ TH

C'est la narration, en quelques paragraphes, de plusieurs journées, mois ou années d'existence sans détails d'action ou de parole.

- l'ellipse, où le temps du récit est infiniment moins important que le temps de l'histoire :

TR $<\infty$ TH

C'est lorsqu'une partie de l'histoire est passée sous silence ${ }^{27}$.

Au cinéma, il faut différencier la durée de la séquence et la durée du film.

\section{1 - Durée de la séquence}

Généralement au cinéma, si l'on prend comme unité la séquence, elle respecte souvent la chronométrie des actions qu'elle montre : l'événement raconté dure dans la diégèse aussi longtemps que le temps qu'il faut pour le raconter.

C'est le cas dans notre film, où pour la plupart des séquences, le temps du récit équivaut au temps de l'histoire.

Il s'agit donc de scènes filmiques.

Prenons l'exemple de la séquence 14, où Santiago Nasar, Cristóbal Bedoya, ainsi qu'une amie à eux, venus accueillir l'évêque, sont sur le quai : nous y voyons la colère de Santiago, car l'évêque ne s'est même pas arrêté au village, et une, discussion à propos du festin de la veille, à l'occasion du mariage d'Ángela.

La durée diégétique est strictement identique à la durée narrative.

109 D'autre part, notre film nous offre un cas de ralenti : il s'agit de la séquence du rêve de Santiago (séq. 4). Cette technique cinématographique a été choisie pour représenter le subconscient du personnage. De plus, les bruits de fond qui accompagnent cette séquence sont les battements de cœur de ce dernier.

110 Nous avons également dans Crónica... un parfait exemple d'ellipse entre les séquences 67 et 68 .

111 A la séquence 67, nous voyons Ángela, encore jeune femme, écrire une lettre d'amour à Bayardo San Román.

112 A la séquence 68 , nous la voyons à nouveau en train d'écrire, mais cette fois-ci, elle a beaucoup vieilli : ses cheveux gris et ses traits tirés nous permettent donc d'affirmer 
qu'il s'agit d'une ellipse, puisqu'on a une suppression temporelle qui intervient entre deux séquences. Le temps du récit est infiniment moins important que le temps de l'histoire. Cette ellipse nous, permet de comprendre que les sentiments amoureux qu'éprouve Ángela envers Bayardo, après le départ de celui-ci, resteront intacts pendant de nombreuses années, ce qui déjà laisse entrevoir le dénouement de l'histoire. En ce qui concerne le récit sommaire, les exemples sont relativement rares au niveau de la séquence. Cependant, nous en avons relevé un à la première séquence, lorsque Cristóbal Bedoya, 27 ans après le crime, arrive au village. Le bateau sur lequel il navigue passe devant la maison des Nasar, et il raconte que c'était un ancien entrepôt racheté par le père de Santiago pour en faire un magasin d'importation, puis transformé en maison.

114 Il raconte donc en quelques mots ce qui s'est passé en plusieurs mois, voire plusieurs années :

«La casa de Santiago Nasar había sida un antiguo depósito de dos pisos. Su padre lo compró para poner una tienda de importación, y sólo cuando se iba a casar, lo convirtió en una casa para vivir ${ }^{28}$.

Il s'agit bien d'un cas de récit sommaire, puisque le temps du récit est plus court que le temps de l'histoire.

Enfin, dans notre film, nous n'avons remarqué, au niveau de la séquence, aucune pause telle que Genette l'entend.

\section{2 - Durée du film}

117 A l'échelle du film pris dans sa globalité, nous voyons que plus de 27 ans sont racontés en moins de deux heures.

118 Donc le temps du récit est bien plus court que le temps de l'histoire. Ceci est évidemment la configuration la plus courante au cinéma.

\section{C / LA FRÉQUENCE}

119 Qu'est-ce que la fréquence narrative ? C'est le rapport établi entre le nombre de fois que tel ou tel événement se trouve évoqué par le récit, et le nombre de fois qu'il est supposé survenir dans la diégèse ${ }^{29}$.

120 La fréquence est l'un des aspects essentiels de la temporalité narrative. En effet, un événement n'est pas seulement capable de se produire ; il peut aussi se reproduire ou se répéter.

Genette distingue précisément quatre types de relations de fréquence :

1/ Tout d'abord, un récit peut raconter une fois ce qui s'est passé une fois dans la diégèse, d'où la formule suivante $: 1 \mathrm{R} / \mathbf{1 H}$.

Selon Genette, « cette forme de récit, où la singularité de l'énoncé narratif répond à la singularité de l'événement narré, est évidemment de loin la plus courante $»^{30}$.

Il a proposé de l'appeler récit singulatif:

Dans notre film, ce type de récit est présent dans de nombreuses séquences montrant des faits particuliers, par exemple la séquence 32, où Bayardo achète la maison du veuf de Xious, ou la séquence 33 dans laquelle se déroule le mariage d'Ángela et Bayardo. 
Ces deux événements se sont effectivement produits une fois dans la diégèse, et sont racontés une fois dans le récit.

2/ Un récit peut raconter $\mathbf{n}$ fois ce qui s'est passé $\mathrm{n}$ fois dans la diégèse, soit $\mathbf{n R} / \mathbf{n H}$.

En réalité, ce deuxième type de relation est semblable au premier puisque «les répétitions du récit ne font que répondre [...] aux répétitions de l'histoire ${ }^{29}$. Ce type anaphorique reste donc singulatif. Nous l'assimilerons au premier type, représenté par la formule : $1 \mathbf{R} / \mathbf{1 H}$.

Au niveau du film pris dans sa globalité, nous avons remarqué une certaine répétition au sujet de l'intention meurtrière des frères Vicario.

Cette intention est annoncée par divers personnages :

- d'abord à la séquence 14, des jeunes filles du village, parlant à Margot, disent :

" $i$ No te has enterado? Los hermanos Vicario quieren matar a Santiago porque

dicen que deshonró a Ángela $»^{31}$.

-à la séquence suivante (séq. 15), c'est Margot qui avertit à son tour sa mère :

«El marido de Angela Vicario descubrió, que ella no era virgen, y la devolvió a su

casa... y los hermanos de Angela dicen que Santiago es el responsable, y por eso quieren matarle $\aleph^{32}$.

-à la séquence 42 , les frères Vicario eux-mêmes annoncent leur intention au boucher du village :

"Vamos a matar a Santiago »",

puis à Clotilda Armenta, la propriétaire d'une boutique, à la séquence 44 ,

«Le estamos buscando para matarle $»^{34}$.

-à la séquence 46 , Pornoy le policier dit au maire :

«Los hermanos Vicario están diciendo a todo el mundo que quieren matar a

Santiago Nasar ${ }^{35}$.

-à la séquence 47, Clotilda Armenta dit à un enfant de chœur :

« Acércate, ve a decirle al padre Amador que los hermanos Vicario quieren matar a

Santiago Nasar $"{ }^{36}$.

puis à la séquence 53 , elle dit à une mendiante :

"Dile a la cocinera de la casa Nasar que van a mater a Santiago, corre " $^{37}$

-à la séquence 55, Cristóbal Bedoya dit à la cuisinière des Nasar :

" $i$ Dónde está Santiago ? [...] Es urgente, le buscan para matarle $»^{38}$.

- à la séquence 57, les frères Vicario disent à Cristóbal Bedoya :

«Cristóbal Bedoya, dile a Santiago Nasar que le estamos esperando para matarle »,

lequel dit au colonel :

«Los hermanos Vicario van armados con cuchillos para mater a Santiago Nasar »"

-à la séquence 58, le père de Flora, la fiancée de Santiago, dit à ce dernier :

«Los hermanos Vicario te están buscando para matarte »"

- enfin, à la séquence 61 , la foule crie à Santiago :

«Santiago, te van a matar, corre Santiago, que te van a matar... »"

Dans ce cas, les répétitions dans le récit ne font que refléter les répétitions dans l'histoire. Il nous a semblé intéressant de faire ce relevé exhaustif afin de montrer, à travers ce procédé de répétition, que la rumeur avait alerté le village entier, et de poser la question suivante : comment malgré tout le crime n'a-t-il pu être évité ?

3 / Un récit peut raconter $n$ fois ce qui ne s'est passé qu'une fois dans la diégèse, soit $\mathrm{nR} / \mathbf{1 H}$.

Dans ce type de récit, « les récurrences de l'énoncé ne répondent à aucune récurrence d'événements ${ }^{43}$. 

images. nH. de l'habitude de Santiago : de la almohada" 46 .

Au cinéma, le récit verbal tient une place aussi importante que l'image. La polyphonie des matières de l'expression rend donc la fréquence plus complexe que dans le roman. En effet, un même événement peut être raconté tantôt par des mots, tantôt par des

L'un des exemples les plus significatifs se trouve aux séquences 41 et 42 . A la séquence 42 , nous voyons les frères Vicario venus chez le boucher pour aiguiser leurs couteaux.

A la séquence 41 , le boucher raconte au juge ce même événement. Nous avons donc un même événement raconté d'une part à l'aide d'images, d'autre part à l'aide de mots.

Il s'agit donc d'un récit répétitif.

4 / Enfin, un récit peut raconter une fois ce qui s'est passé $\mathrm{n}$ fois dans la diégèse : $\mathbf{1 R}$ /

C'est un type de récit «où une seule émission narrative assume ensemble plusieurs occurrences du même événement $»^{44}$.

Genette propose de l'appeler récit itératif (du latin iterare signifiant recommencer).

L'image ne disposant pas de temps grammaticaux, il n'est pas facile d'exprimer qu'une action montrée une seule fois à l'écran vaut pour plusieurs actions similaires. Souvent, c'est le jeu de l'acteur (mécanique ou automatique) ou le dialogue, qui permettent de comprendre que le geste que nous voyons est arrivé plusieurs fois ${ }^{45}$.

Dans notre film, ce sont les dialogues ou la narration de Cristóbal Bedoya qui nous donnent des indications de fréquence montrant le caractère itératif d'une action. Ainsi à la séquence 5, l'adverbe "toujours " ("siempre") nous montre le caractère quotidien

"Santiago siempre dormía como durmió su padre, con el revólver escondido debajo

De plus, cet imparfait de répétition "dormait » (“dormía”) nous raconte non ce qui s'est passé, mais ce qui se passait régulièrement.

5 A la séquence 69, le maire et Cristóbal Bedoya voient Ángela se diriger vers la poste pour y déposer une lettre. Le caractère itératif de l'action est confirmé par l'indication de fréquence " par semaine » ("por semana") :

"Desde entonces, no ha dejado de escribirle... Una o dos cartas por semana" 47 .

Cependant, ce type de récit n'est que rarement employé dans notre film, lors d'actions ponctuelles comme nous venons de voir.

A travers cette analyse, nous avons pu nous rendre compte que dans notre récit, le temps est totalement disloqué.

Nous nous sommes alors demandé pourquoi le temps est manipulé d'une telle façon.

Lorsque nous retrouvons une telle manipulation du temps dans des récits littéraires ou cinématographiques, l'intention de l'auteur ou du réalisateur est généralement de donner du suspense au lecteur ou au spectateur. Mais dans Crónica de una muerte anunciada, il n'y a aucun suspense : le titre-même nous annonce qu'un des personnages va mourir; à la séquence 3 , nous apprenons que c'est Santiago Nasar qui est mort à l'âge de 21 ans; enfin, à la séquence 14, nous découvrons qui sont les responsables du crime, et pourquoi ils ont tué Santiago. 

plusieurs raisons : point d'incompréhension. des personnages. passer?».

Si le temps du récit est fragmenté et non chronologique dans notre film, c'est pour

- tout d'abord, l'intention de ce genre de récit sans surprise, dénué de suspense, est de concentrer l'intérêt sur la psychologie des personnages.

Effectivement, on retourne vers le passé pour exposer les causes des faits présents. Par conséquent, la suite des événements n'est plus directement temporelle, mais causale. Ainsi, la chronologie des événements est bouleversée, et restructurée en fonction d'un point de vue subjectif. Les analepses créent donc une temporalité spécifique, qui permet d'introduire un récit subjectif à la première personne. Or, ce type de récit est tout à fait propice à l'exploration des domaines psychologiques.

Grâce à l'ordre des événements tels qu'ils apparaissent dans ce récit cinématographique, nous parvenons à comprendre parfaitement la psychologie des personnages du récit premier (principalement Cristóbal Bedoya et la mère de Santiago), qui est sans cesse complété par des récits seconds.

La structure du récit est telle qu'elle parvient, par une série d'analepses, à mettre l'accent sur l'émotion et les sentiments des personnages.

D'une part, en ce qui concerne Cristóbal Bedoya, le film est en quelque sorte la matérialisation de son souvenir : il revit donc les circonstances qui l'ont amené à ce

D'autre part, nous donnerons un exemple, le plus significatif à nos yeux, qui explique l'état d'esprit de la mère de Santiago. Il s'agit des séquences 61 et 62 . A la séquence 61, Santiago se trouve sur la place ; poursuivi par ses agresseurs, il court vers sa maison. $\mathrm{Au}$ moment où il s'apprête à y entrer, la porte se ferme devant lui. Le début de la séquence 62 coïncide avec la fin de la séquence 61, puisqu'on voit une porte claquer. Seulement 27 ans ont passé, et la mère de Santiago répète la même action que le jour de la mort de son fils, afin d'expliquer ce geste à Cristóbal Bedoya venu lui rendre visite.

Ce hiatus temporel a énormément d'impact sur le spectateur: il lui permet de comprendre parfaitement le sentiment de culpabilité de cette mère, ainsi que sa consternation et son profond désespoir.

De plus, nous nous apercevons que cette femme revit ce terrible moment comme s'il avait eu lieu la veille, alors que près de 30 ans se sont écoulés.

Par conséquent, la structure du récit telle qu'elle se présente est d'un point de vue psychologique beaucoup plus percutante que si l'ordre des événements avait été chronologique, dans la mesure où elle permet de comprendre exactement l'état d'esprit

- ensuite, ce type de récit a pour but de susciter la curiosité du spectateur. Effectivement, dans notre film, le spectateur prend part au récit en s'interrogeant, en se posant des questions. Il ne se demande pas : « que va-t-il se passer? » puisqu'il le sait, mais « comment cela va-t-il se

Il est curieux par exemple de savoir dans quelles circonstances exactes va se dérouler le crime. Et ce n'est qu'à la fin du film que ces circonstances lui seront dévoilées par le biais de la cinquième analepse.

Les analepses internes complétives ont donc pour but dans notre film de satisfaire la curiosité du spectateur, en complétant un manque ou une omission volontaire, en comblant une lacune du récit que Genette appelle paralipse ${ }^{48}$. 
161 Les autres analepses, c'est-à-dire les analepses externes, expliquent ou éclairent certains événements, en dévoilant des faits nécessaires à la compréhension de la diégèse.

Par exemple, les deuxième et troisième analepses nous relatent la préparation du crime et nous permettent de comprendre comment un tel drame n'a pas pu être évité.

- de plus, le fait pour le spectateur de savoir dès le début du film que l'histoire finira de façon tragique, introduit une atmosphère de fatalité irrémédiable, qui contribue beaucoup à donner au film son caractère si envoûtant.

D'ailleurs, cette construction existait déjà à propos de la tragédie antique: le dénouement fatal de l'histoire d'œdipe ou d'Antigone est connu d'avance, et le drame n'en suscite pas moins la terreur et la curiosité des spectateurs.

D'autre part, nous nous sommes demandés ce qui déclenchait les analepses dans Crónica...

Dans notre film, le souvenir obéit à la fois aux caprices de la mémoire et au hasard des circonstances.

Ce sont souvent des photos qui déclenchent le souvenir : la photo de Santiago sur sa tombe (séq. 3) est le point de départ de la première analepse; la photo de tous les habitants réunis sur le quai (séq. 13) évoque le souvenir du passage de l'évêque au village ; la photo d'Ángela (séq. 38) et celle du père Amador (séq. 48) que Cristóbal Bedoya retrouve à l'édifice colonial en même temps que le dossier sur l'enquête, déclenchent le souvenir du procès.

167 Parfois, les lieux sont également évocateurs du passé :

- la chambre de Bayardo et Ángela dans laquelle se retrouve cette dernière quelque temps après le crime (séq. 65), fait naître en elle le souvenir de la nuit de noces.

- le fleuve lui aussi est un témoin du passé : lorsque Cristóbal Bedoya se trouve assis au bord du fleuve (séq. 17), il se souvient de l'arrivée au village de Bayardo San Román, par ce même fleuve.

168 Enfin, nous achèverons cette étude en évoquant le fleuve, qui est un élément spatial important, dans la mesure où il symbolise le temps. Effectivement le fleuve a une dimension temporelle. C'est pourquoi sa présence est tout à fait significative dans notre film: d'une part, il est témoin du passé, d'autre part, l'écoulement de l'eau reflète l'écoulement du temps, le rythme de la vie.

169 Nous avons donc essayé d'analyser le temps dans notre film, en mettant en relief toutes les distorsions temporelles présentes.

170 Notre récit cinématographique étant le récit du souvenir, l'anachronisme est l'une de ses principales caractéristiques. En effet, le souvenir obéit à d'autres lois que celle du temps. Comme le dit Proust,

« Notre mémoire ne nous présente pas d'habitude nos souvenirs dans leur suite chronologique, mais comme un reflet où l'ordre des parties est inversé $»^{49}$.

171 Nous ajouterons que si le temps est ainsi disloqué, peut-être est-ce par souci de vraisemblance. La structure du récit reflète sans doute les méandres de la mémoire. La mémoire joue également un rôle très important sur les variations de rythme.

De façon plus générale, nous pouvons dire que la mémoire altère profondément la notion de temps. 


\section{NOTES}

1. Cf. Christian METZ : Essai sur la signification au cinéma, Klincksieck, Paris, 1968, p. 27.

2. Pour caractériser cet univers construit par le film, Souriau a proposé le terme de diégèse qu'il définit ainsi : « Tout ce qui appartient dans l'intelligibilité à l'histoire racontée au monde proposé ou supposé par la fiction ». Etienne SOURIAU : L'univers filmique, Flammarion, Paris, 1953.

3. Cf. Gérard GENETTE : Figures III, Éditions du Seuil, Paris, 1972, p. 78.

4. Cf. André GAUDREAULT et François JOST : CINÉMA ET RÉCIT II, Le Récit Cinématographique, Nathan, 1990, p. 104.

5. Cf. Gérard LAVERGNE : "Terminologie narratologique. Essai d'éclaicissement » in "Temps et récit romanesque», actes du 2e Colloque International du Centre de Narratologie Appliquée, Université de Nice, 1991 p. 191.

6. «Mais il semblait heureux avec son père, jusqu'au jour où celui ci mourut subitement, trois ans plus tôt ».

7. « le lundi de sa mort»

8. «Six heures vingt-cinq ».

9. «Six mois avant que Santiago Nasar ne soit tué, un étranger arriva dans notre village... Il s'appelait Bayardo San Román. »

10. "Je suis revenu 27 ans après dans ce village oublié, pour essayer de recomposer avec des éclats épars le miroir cassé de la mémoire ».

11. Cf. Jacques AUMONT et Michel MARIE : L'analyse des films, Nathan, 1989. "Une séquence (= suite de plans) est une unité narrative et spatio-temporelle », p. 36.

12. Cf. André GAUDREAUT et François JOST : CINÉMA ET RÉCIT II, Le Récit Cinématographique, p. 105.

13. Gérard GENETTE : op. cit., p.79 - 82.

14. Idem : p. 90.

15. Idem : p. 89.

16. Idem : p. 90- 92.

17. « Ils l'ont tué... Il est mort».

18. « Envoie-moi Divina Flor pour qu'elle me réveille à 5 h30 »

19. « 6 mois avant que Santiago Nasar ne soit tué, un étranger arriva dans notre village »

20. Gérard GENETTE : Figures 111, p 101.

21. Marcel MARTIN : Le langage cinématographique. Les éditions du Cerf, Paris, 1994 (sixième édition), p. 182.

22. Idem : p. 92 - 95.

23. Idem : p. 92 - 95.

24. «Ce fut lui »

25. Cf. Étienne SOURIAU : L'univers filmique, Flammarion, Paris, 1953.

26. Cf. Gérard GENETTE : Figures III, p. 123.

27. Idem : p. 129.

28. «La maison de Santiago Nasar avait été un ancien entrepôt à deux étages. Son père l'acheta afin d'y établir un magasin d'importation, et ce n'est qu'au moment de son mariage qu'il le transforma en maison d'habitation ».

29. Cf. André GAUDREAULT et François JOST : CINÉMA ET RÉCIT II, Le Récit Cinématographique, p. 121.

30. Gérard GENETTE : Figures III, p. 146.

31. « Tu n'es pas au courant? Les frères Vicario veulent tuer Santiago parce qu'il a deshonoré Ángela ». 
32. « Le mari d'Ángela Vicario a découvert qu'elle n'était pas vierge, et il l'a ramenée chez elle... et les frères Vicario disent que Santiago est le responsable, et c'est pour cela qu'ils veulent le tuer. »

33. « Nous allons tuer Santiago Nasar»

34. « Nous sommes en train de le chercher pour le tuer».

35. « Les frères Vicario disent à tout le monde qu'ils veulent tuer Santiago Nasar ».

36. «Approche-toi. Va dire au père Amador que les frères Vicario veulent tuer Santiago Nasar ».

37. « Dis à la cuisinière des Nasar qu'on va tuer Santiago, cours».

38. "Où est Santiago ? C'est urgent, on le cherche pour le tuer».

39. «Cristóbal Bedoya, dis à Santiago Nasar que nous l'attendons pour le tuer ».

40. «Les frères Vicario sont armés de couteaux et ont l'intention de tuer Santiago Nasar ».

41. «Les frères Vicario te cherchent pour te tuer".

42. "Santiago, ils vont te tuer, cours Santiago, ils vont te tuer".

43. Gérard GENETTE : Figures III, p. 147.

44. Idem : p. 148.

45. Cf. André GAUDREAULT et François JOST : CINÉMA ET RÉCIT II, Le Récit Cinématographique, p. 123.

46. «Santiago dormait toujours comme son père avait dormi, le révolver caché sous l'oreiller ».

47. « Dès lors, elle n'a cessé de lui écrire... Une ou deux lettres par semaine ».

48. Gérard GENETTE : op. cit., p. 93.

49. Marcel PROUST : A la recherche du temps perdu, Collection de la Pléiade, Gallimard, t. I, nov. 55, p. 578.

\section{AUTEUR}

\section{SOPHIE VELASCO}

Université de Nice-Sophia Antipolis 